\title{
Design of Highly Birefringent Holey Fibers with Near-Zero Ultra-flattened Chromatic Dispersion and Ultralow Confinement Loss
}

\author{
M. Samiul Habib ${ }^{*}$, M. Selim Habib \\ Dept. of EEE, Rajshahi University of Engineering \& Technology, Rajshahi-6204. \\ Email: "samiul.engieee@gmail.com \\ S. M. A. Razzak, M. A. Goffar Khan \\ Dept. of EEE, Rajshahi University of Engineering \& Technology, Rajshahi-6204. \\ Y. Namihira and M. A. Hossain \\ Graduate School of Engineering and Science, University of the Ryukyus, 1 Senbaru, Nishihara, Okinawa \\ 903-0213, Japan.
}

\begin{abstract}
We present a holey fiber (HF) with elliptical air-holes located in the center core area that ensures high birefringence, near-zero ultra-flattened chromatic dispersion and very low confinement losses in a wide wavelength range. The finite element method with perfectly matched boundary layer is used to investigate the guiding properties. It is demonstrated that it is possible to design a low-loss dispersion-flattened HF with a high birefringence of 0.0033 at a $1.55 \mu \mathrm{m}$ wavelength. According to simulation, near-zero ultra-flattened dispersion of $0 \pm 0.5 \mathrm{ps} / \mathrm{nm} / \mathrm{km}$ is obtained in a 1.25 to $1.65 \mu \mathrm{m}$ wavelength range with low confinement losses of $0.0008 \mathrm{~dB} / \mathrm{km}$ in the entire band of interest.
\end{abstract}

Index Terms - Holey fiber, birefringence, chromatic dispersion, confinement loss.

\section{INTRODUCTION}

Index guiding photonic crystal fibers (PCFs) or holey fibers [1] usually consist of a hexagonal arrangement of microscopic air-channels running down the length of the silica based fiber surrounding a central solid silica core. Holey fibers (HFs) guide the light via one of two mechanisms: effective-index guidance and photonic-bandgap (PBG) guidance. In the HFs with effective-index mechanism, the light is guided based on the total internal reflection between a solid core and a cladding region with multiple air-holes [1]. On the other hand, HFs based on PBG has the capability to control the guidance of light within a certain frequency band [2]-[3]. Holey fibers have drawn increased attention nowadays because of many of their attractive properties [4]; for example, very high or very low nonlinearity, wideband dispersion-flattened characteristics, high birefringence, endlessly single mode guiding, and many others.

By modulating the parameters of the holey cladding, it is possible to design application specific guiding properties [5]. In this study, we have proposed a birefringent HFs, comprising of hexagonal lattice of air holes, where four elliptical air-holes are inserted in the center core area (two elliptical air- 
holes in first ring and second ring also contains two elliptical air-holes) to achieve highly birefringent fibers ensuring with flattened dispersion in the entire band of interest.

Birefringence is usually an undesirable property of fiber optics. In many sensing applications and in applications where light is required to maintain a linear polarization state, a high level of birefringence is often required [6]. Birefringent HFs can simply be realised [8] compared to conventional fibers, since the refractive index contrast between the core and the cladding is higher than the refractive index contrast of conventional fibers. The key point in realizing the birefringence is to destroy the symmetry of the fiber structure and increase the effective index difference between the two orthogonal polarization modes [6]. The structural symmetry can be destroyed either by altering the air hole sizes near the core area [7]-[8], or by distorting the shape of the air holes (elliptical air holes) [9]. There are a number of ways of designing birefringence in HFs. One common technique is to break the HFs structural symmetry by inserting elliptical air hole along two orthogonal axes near the core region. This results in increasing the difference between the effective indexes of the orthogonal polarization modes. For many applications it is essential to design HFs that exhibit simultaneous high birefringence, low confinement losses, small effective area, low and flattened chromatic dispersion in a wide wavelength range. In broadband communications systems, fiber dispersion and confinement loss play very important roles. For example, in wavelength division multiplexing systems it is essential to maintain a uniform response in different wavelength channels. This is strictly achieved by ensuring ultra-flattened dispersion characteristics of fibers [10]. This novel property of HFs helps in tuning transmission characteristics namely dispersion, nonlinearity, and confinement loss in smart ways.

\section{DESIGN METHODOLOGY}

Fig. 1 shows a simple geometry of the proposed highly birefringence dispersion flat holey fiber (HBDF-HF). The air-hole diameters on the first ring and second ring is $d_{1}, d_{2}$, while air-hole diameters on the $3^{\text {rd }}$ to $5^{\text {th }}$ ring is same $d$ and diameter of elliptical air-holes on first ring and second ring in x direction is $d_{\mathrm{x}}$ and in y direction is $d_{\mathrm{y}}$. The air-hole pitch is $\Lambda$. The refractive index of the air hole and fiber silica is $n_{\mathrm{a}}=1$ and $n_{\mathrm{s}}=1.45$ respectively. As shown in Fig. 1, there are three degrees of freedom, namely $d_{1}, d_{2}$ and $\Lambda$ for controlling the dispersion nature of the five-ring HF. Dimension of the first ring is scaled down to flatten the dispersion characteristics. Birefringence can be achieved over creating asymmetry, either by alerting the air hole size or distorting the shape of the air holes into elliptical size in the core area. In Fig. 1 the background material is pure silica surrounding with airholes. Air-holes in the cladding are arranged in a hexagonal symmetry with a common pitch $\Lambda$. 


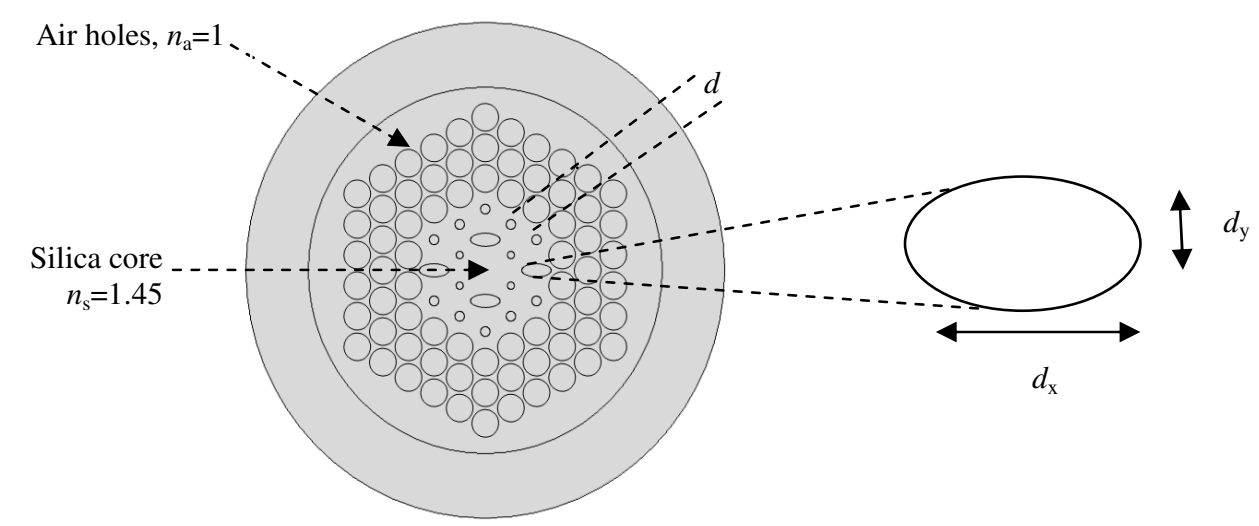

Fig. 1 Geometry of proposed five-ring HBDF-HF with $1^{\text {st }}$ ring air-hole diameter $d_{1}, 2^{\text {nd }}$ ring air-hole diameter $d_{2}, 3^{\text {rd }}$ to $5^{\text {th }}$ ring diameter is same $d$, diameter of elliptical air-holes on first ring and second ring in $\mathrm{x}$ direction (major axis) is $d_{\mathrm{x}}$ and in y direction (minor axis) is $d_{y}$, air-hole pitch $\Lambda$ and number of ring, $\mathrm{Nr}=5$.

\section{Simulation Methodology AND EQUATION}

The COMSOL software 4.2 version is used as a simulation tool. The finite-element method with perfectly matched boundary layers (PML) is used to calculate effective refractive index, birefringence, chromatic dispersion, and confinement loss of HFs. The FEM directly solves the Maxwell equations to best approximate the value of the effective refractive index. Once the modal effective refractive index, $n_{\text {eff }}$ is obtained by solving an eigen value problem drawn from Maxwell equations using the COMSOL software 4.2, birefringence, chromatic dispersion, and confinement loss of HFs can be easily calculated.

\section{Chromatic Dispersion}

Chromatic dispersion is one of the most important modal properties of the HFs. Chromatic dispersion is composed of material and waveguide dispersion. Control of chromatic dispersion is very crucial for practical applications to optical fiber communication system. Chromatic dispersion $D$ can be obtained using the following relation [11]

$$
D(\lambda)=-\frac{\lambda}{c} \frac{d^{2} \operatorname{Re}\left[n_{e f f}\right]}{d \lambda^{2}}
$$

where $\operatorname{Re}\left[n_{\text {eff }}\right]$ is the real part of effective refractive index $n_{\text {eff }}, \lambda$ is the wavelength, $\mathrm{c}$ is the velocity of light in vacuum. The material dispersion given by Sellmeier formula is directly included in the calculation. Therefore, $D$ in (1) corresponds to the chromatic dispersion of the HF.

\section{Confinement Loss}

The confinement loss is a phenomenon whereby part of the guided light penetrates to the cladding region. Due to similar refractive index between core and cladding optical field may penetrate into the 
cladding region results confinement loss. Actually number of air holes in the cladding region limits the confinement loss.

The confinement loss $L_{\mathrm{c}}$ is obtained from the imaginary part of refractive index $n_{\text {eff }}$ as follows [10]

$$
L \mathrm{c}=8.686 \times k_{0} \operatorname{Im}\left[n_{\mathrm{eff}}\right] \times 10^{3} \mathrm{~dB} / \mathrm{km}
$$

where $\operatorname{Im}\left[n_{\text {eff }}\right]$ is the imaginary part of the refractive index, $k_{0}=2 \pi / \lambda$ is the wave number in the free space.

\section{Birefringence and Beat Length}

Birefringent HFs can be achieved by having asymmetric core. This increases the effective index difference between the two orthogonal polarization modes. Birefringence is defined as a difference between effective refractive indices of two fundamental polarization modes [7], [12] and can be written as

$$
B=\left|n_{\mathrm{x}}-n_{\mathrm{y}}\right|
$$

Where $n_{\mathrm{x}}$ and $n_{\mathrm{y}}$ are the effective refractive indices of each fundamental mode.

The beat length $L_{\mathrm{B}}$ which can be defined as follows [13]

$$
L_{\mathrm{B}}=\lambda / B
$$

where $\lambda$ is the operating wavelength.

\section{Simulation Results}

In this proposed design, air -hole diameter of the inner ring and hole-to-hole spacing effects, on the birefringence, chromatic dispersion and confinement losses are carefully investigated. Optimizing the geometrical parameters, such as air-hole diameters and pitch $\Lambda$, the ultra-flattened chromatic dispersion with high birefringence HF can be efficiently designed. In our simulation we first set airhole diameters of the outer cladding at $d / \Lambda=0.9$. In the outer most cladding a high value normalized diameter is chosen for better field confinement. Dimension of the first ring is lowered down to flatten the dispersion characteristics. As shown in Fig. 1, there are three modest number of tuning parameters namely $d_{1}, d_{2}$ and $\Lambda$ for shaping the dispersion behavior of the five-ring HF. At shorter operating wavelengths, mode is more confined in the core region than at longer wavelengths, and the chromatic dispersion is directly affected from the inner air-hole ring and the air hole arrangements but also from the outer ring air hole sizes and hole-to-hole spacing $\Lambda$. 
Fig. 2 (a) shows an example of the effect of changing $d_{1} / \Lambda$ on the dispersion slope for x polarization with $d_{1} / \Lambda=0.22,0.24$, and 0.26 respectively, for a fixed air-hole pitch $\Lambda=1.42 \mu \mathrm{m}$, outer ring normalized air-hole diameter $d / \Lambda=0.9$ and $d_{\mathrm{x}}=1.42 \mu \mathrm{m}, d_{\mathrm{y}}=0.608 \mu \mathrm{m}$.

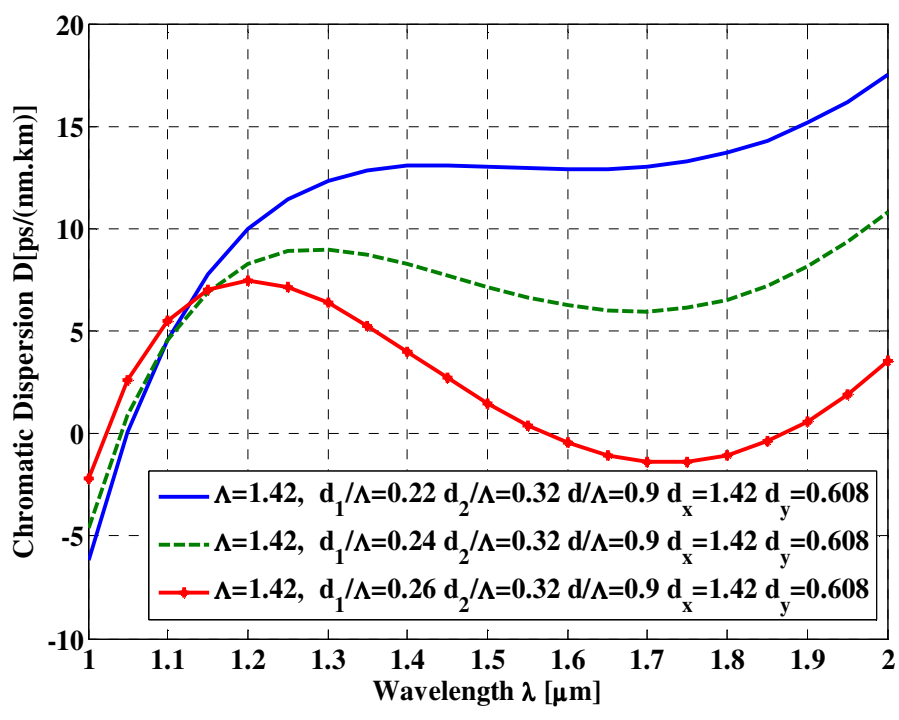

(a)

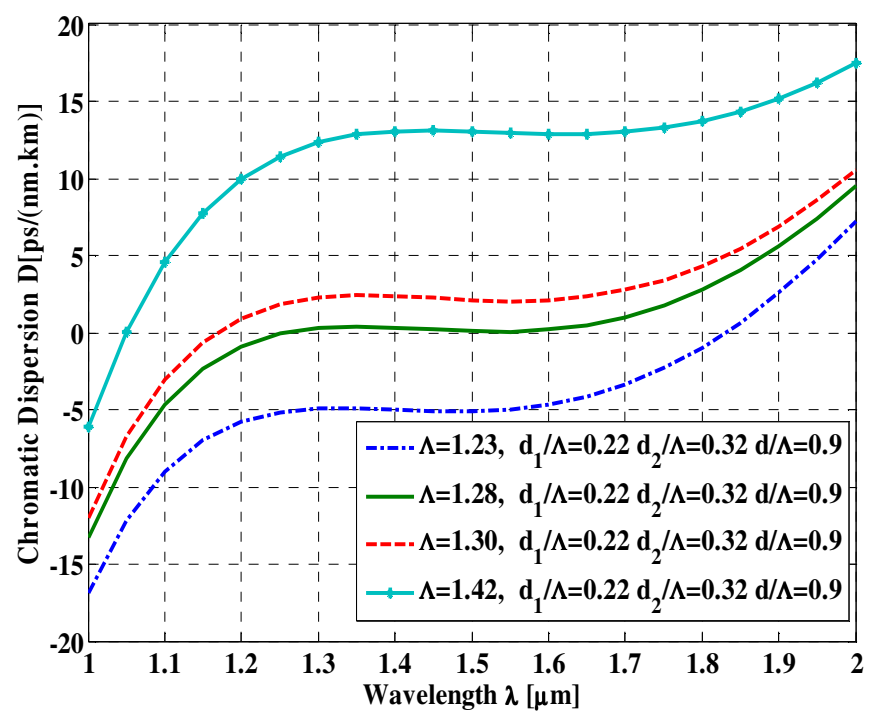

(b)

Fig. 2 (a) Effect of $d_{1} / \Lambda$ on the dispersion slope for x polarization with $d_{1} / \Lambda=0.22,0.24$ and 0.26 respectively, outer ring normalized diameter $d / \Lambda=0.9, d_{2} / \Lambda=0.32 d_{\mathrm{x}} / \Lambda=1$ and $d_{\mathrm{y}} / \Lambda=0.428$ and fixed air-hole pitch $\Lambda=1.42 \mu \mathrm{m}$.

(b) Effect of changing $\Lambda$ on the dispersion level for x polarization with $d_{1} / \Lambda=0.22, d_{2} / \Lambda=0.32, d / \Lambda=0.9, d_{\mathrm{x}} / \Lambda=1$ and $d_{y} / \Lambda=0.428$.

From Fig. 2 (a) it has been seen that flattened dispersion is greatly depend on first ring normalized air-hole diameter $d_{1} / \Lambda$. The dispersion is flat as $d_{1} / \Lambda=0.22$ but the dispersion slope changes significantly from the positive slope to negative one near the wavelength at $1.23 \mu \mathrm{m}$ as $d_{1} / \Lambda=0.24$ and $d_{1} / \Lambda=0.26$.

Fig. 2 (b) shows effect of changing pitch, $\Lambda$ on the dispersion behavior. From designing point of view, a large value of $d / \Lambda$ is chosen for better field confinement. Simulation result reveals that the airhole pitch, $\Lambda$ dominantly influence the dispersion level but insignificant effect on the slope of the dispersion. 


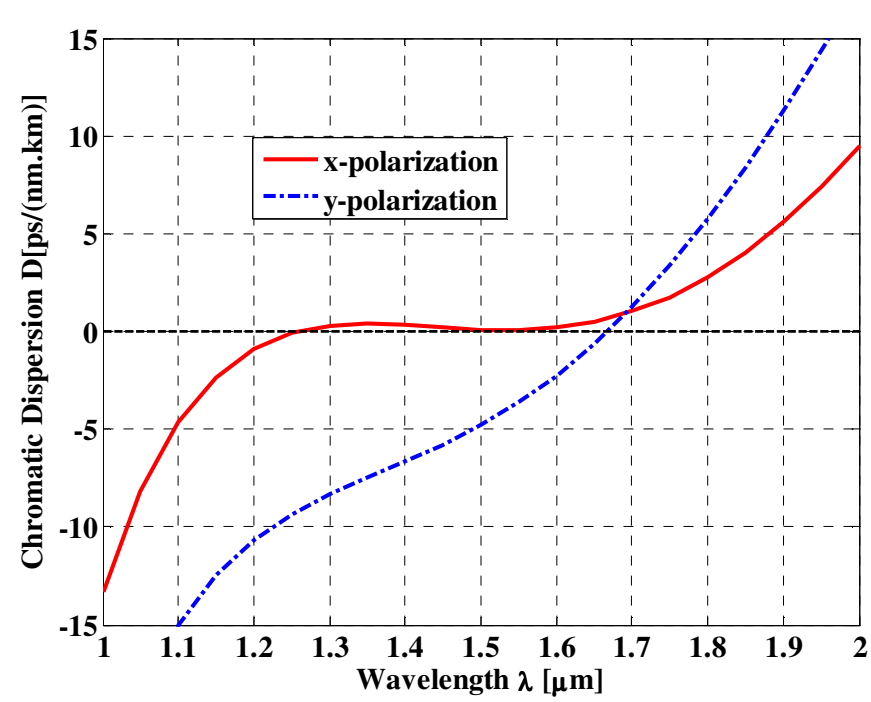

(a)

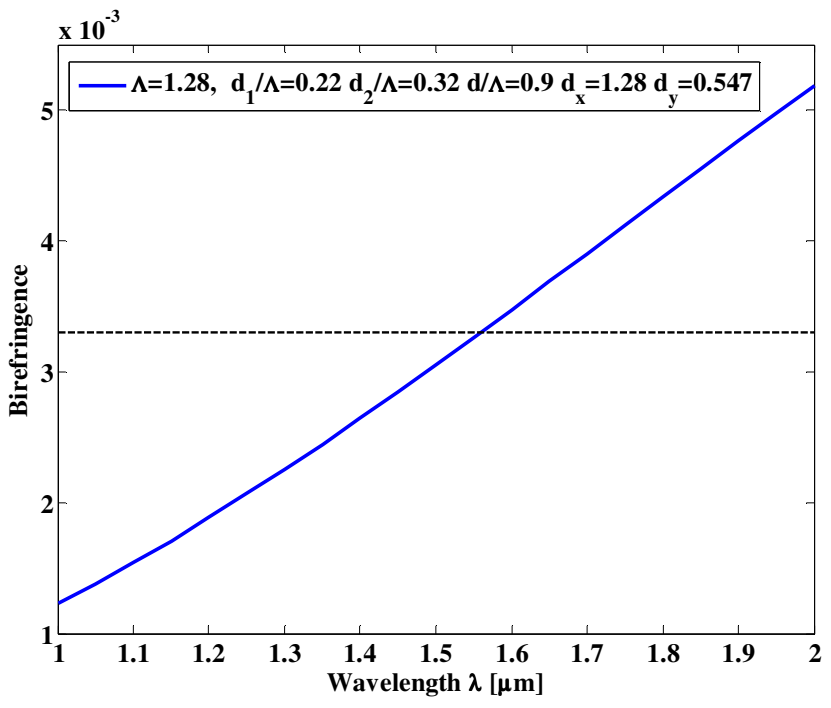

(b)

Fig. 3 (a) Optimum dispersion of the proposed HBDF-HF for $\Lambda=1.28, d_{1} / \Lambda=0.22, d_{2} / \Lambda=0.32, d / \Lambda=0.9, d_{\mathrm{x}} / \Lambda=1$ and

$$
d_{\mathrm{y}} / \Lambda=0.428 \text {. }
$$

(b) Wavelength dependence of birefringence of the proposed HBDF-HF for optimum design parameters: $\Lambda=1.28, d_{1} / \Lambda=0.22$,

$$
d_{2} / \Lambda=0.32, d / \Lambda=0.9, d_{\mathrm{x}} / \Lambda=1 \text { and } d_{\mathrm{y}} / \Lambda=0.428 \text {. }
$$

Fig. 3 (a) shows dispersion property of the HBDF-HF for both x-polarization and y-polarization. Optimizing the geometrical parameters, ultra-flattened dispersion of $0 \pm 0.5 \mathrm{ps} / \mathrm{nm} / \mathrm{km}$ is obtained in a $1.25 \mu \mathrm{m}$ to $1.65 \mu \mathrm{m}$ wavelength range ( $400 \mathrm{~nm}$ flat bandwidth) for x-polarization. For optimum result, air-hole diameter on the first ring is scaled down to shape dispersion property while diameter of airholes on outer rings is kept larger to reduce the confinement loss. Dispersion at $1.55 \mu \mathrm{m}$ is as low as $0.072 \mathrm{ps} / \mathrm{nm} / \mathrm{km}$ for x-polarization.

From our simulation result it is seen that, effective index of y-polarization mode is higher than $x-$ polarization mode. Fig. 3 (b) shows variation of the modal birefringence as a function of wavelength with $\Lambda=1.28, d_{1} / \Lambda=0.22, d_{2} / \Lambda=0.32, d / \Lambda=0.9, d_{\mathrm{x}} / \Lambda=1$ and $d_{\mathrm{y}} / \Lambda=0.428$. This proposed design can operate effectively as a single mode fiber with a high birefringence of 0.0033 at a $1.55 \mu \mathrm{m}$ wavelength. In terms of birefringence our proposed HF structure exhibits much better performances than [6], [7] and [13]. 


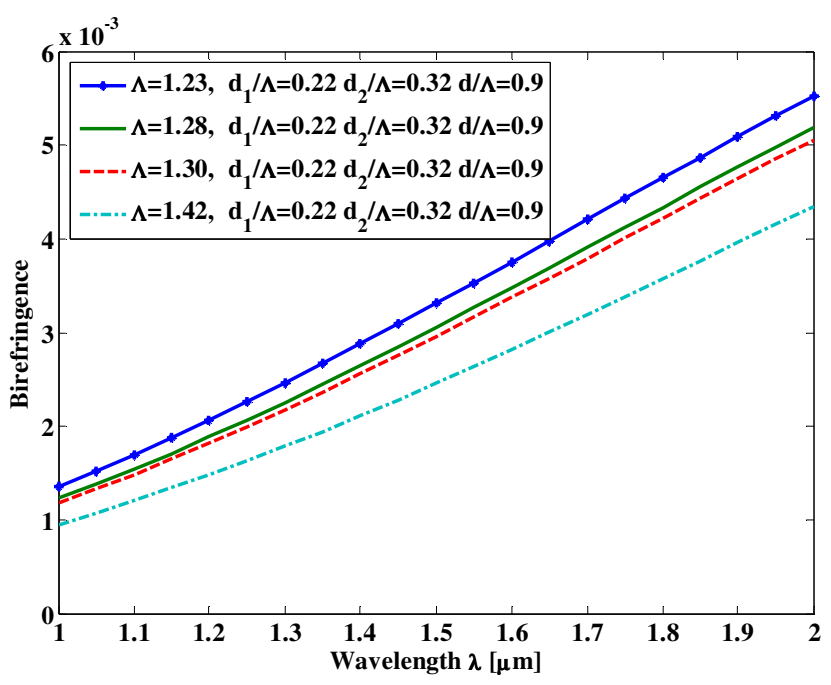

(a)

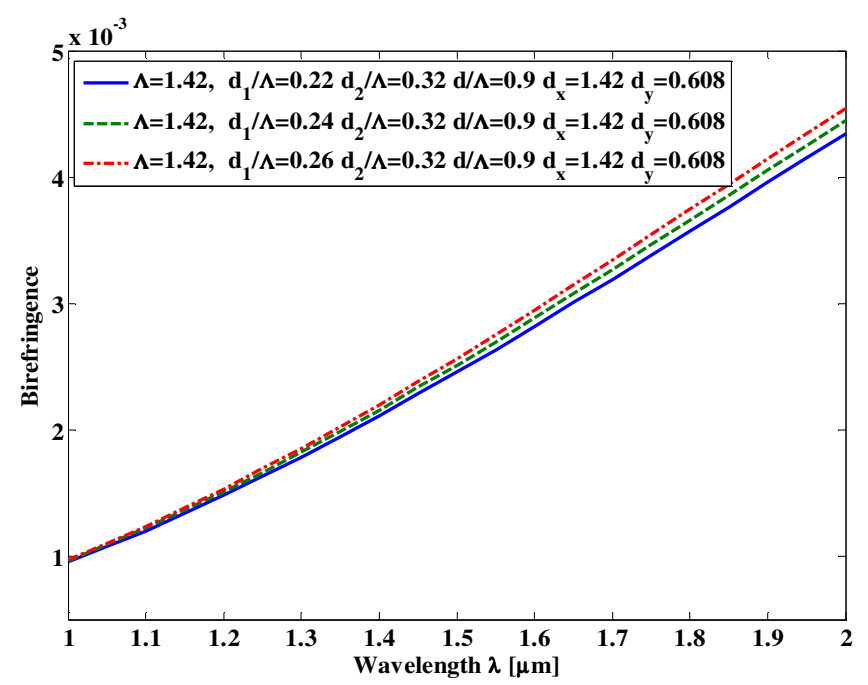

(b)

Fig. 4 (a) Wavelength dependence of birefringence of the proposed HBDF-HF for: $d_{1} / \Lambda=0.22, d_{2} / \Lambda=0.32, d / \Lambda=0.9, d_{\mathrm{x}} / \Lambda=1$ and $d_{\mathrm{y}} / \Lambda=0.428$ and $\Lambda=1.23,1.28,1.30,1.42 \mu \mathrm{m}$.

(b) Wavelength dependence of birefringence of the proposed HBDF-HF for: $d_{2} / \Lambda=0.32, d / \Lambda=0.9, d_{\mathrm{x}} / \Lambda=1$ and $d_{\mathrm{y}} / \Lambda=0.428$, $\Lambda=1.42 \mu \mathrm{m}$ and $d_{1} / \Lambda=0.22,0.24,0.26$.

From Fig. 4 (a) it can clearly seen that, birefringence is sensitive to air-hole pitch. As hole-to-hole spacing decreases, the birefringence increases. So in order to achieve higher birefringence, we decreased the hole-to-hole spacing from $1.42 \mu \mathrm{m}$ to $1.23 \mu \mathrm{m}$.

Our simulations show that there is a tradeoff between low dispersion and birefringence. When the inner-ring air hole size increases the birefringence improves, however, the dispersion slope degrades. From Fig. 4 (b) it is seen than as $d_{1} / \Lambda$ increases birefringence improves but dispersion slope deteriorates as seen from Fig. 2 (a). In Fig. 5 (a), we have plotted the variation of the beat length as a function of the wavelength. Highly birefringence fibers have low beat length. Our proposed design has low beat length of $0.474 \mathrm{~mm}$ at the operating wavelength which is less than reported in [6].

The confinement loss strongly depends on the number of air hole rings, air hole diameter and holeto-hole spacing. In our proposed design, dimension of the outer three rings are kept larger to reduce the confinement loss. Confinement losses of the HF reported in [6], [7] and [13] are very large which is $2.5 \times 10^{-1} \mathrm{~dB} / \mathrm{km}, 1.21 \times 10^{7} \mathrm{~dB} / \mathrm{km}$ and $4.6 \times 10^{6} \mathrm{~dB} / \mathrm{km}$ at the operating wavelength $\lambda=1.55 \mu \mathrm{m}$. 


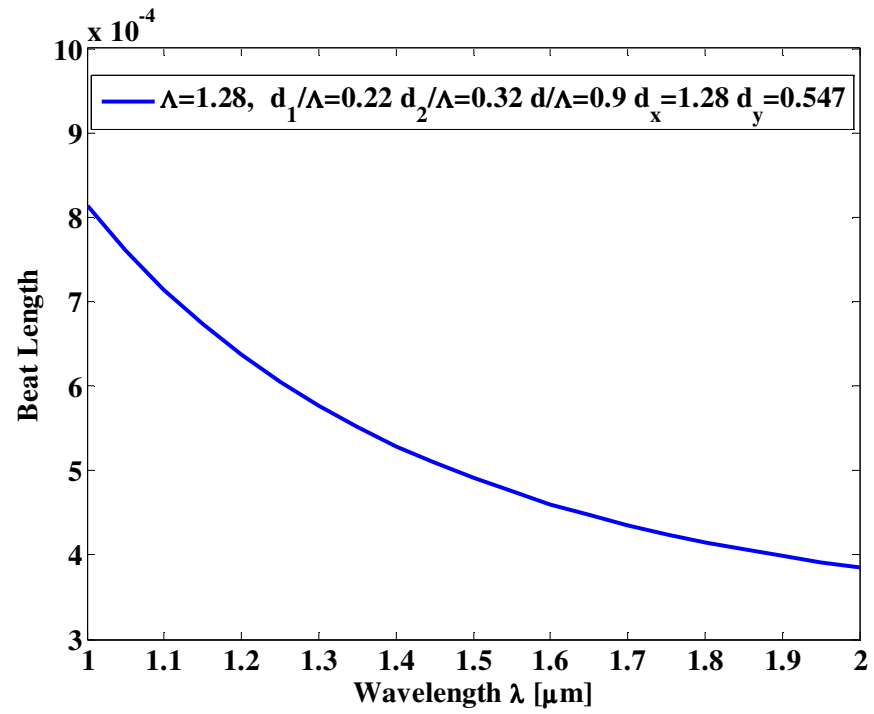

(a)

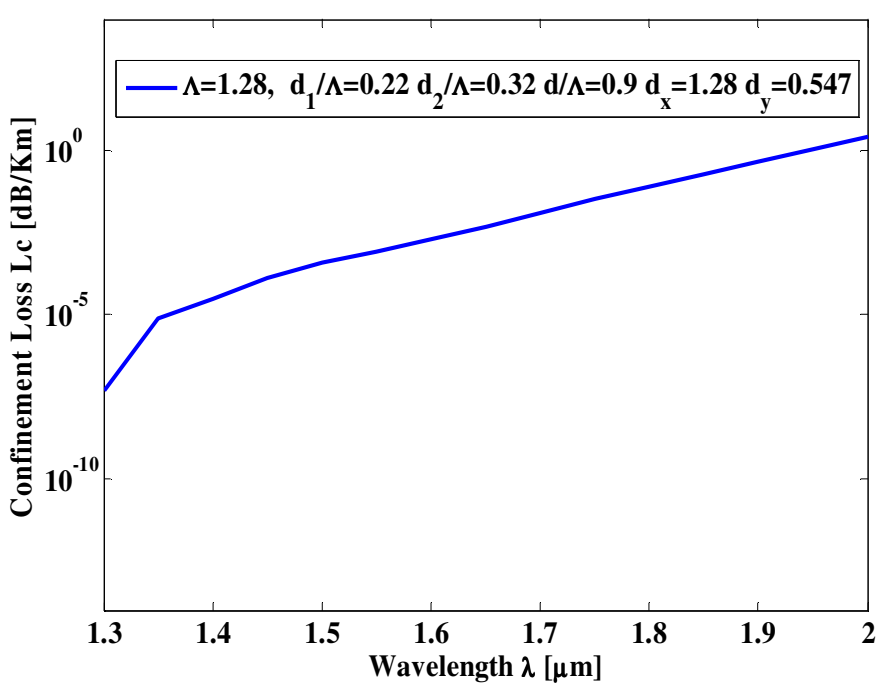

(b)

Fig. 5(a) Beat length of the proposed HBDF-HF for optimum design parameters as a function of wavelength.

(b) Confinement loss of the proposed HBDF-HF for optimum design parameters as a function of wavelength.

This is due to the small number of air-hole rings that have been used in [7] and [13]. However, in our proposed design, confinement loss is $0.0008 \mathrm{~dB} / \mathrm{km}$ at the operating wavelength $\lambda=1.55 \mu \mathrm{m}$. When the diameter of the inner air hole rings is increased, the confinement loss reduced significantly but control of chromatic dispersion is somewhat difficult at shorter wavelength. Fig. 5 (b) shows wavelength dependence of confinement loss for $\mathrm{x}$-polarization. Confinement loss at $1.55 \mu \mathrm{m}$ is as low as $0.0008 \mathrm{~dB} / \mathrm{km}$ for $\mathrm{x}$-polarization.

Therefore, the proposed fiber with a modest number of design parameters, near-zero ultra-flattened dispersion, and leakage properties may pave the way for different applications in optics including optical parametric amplification, wavelength conversion, soliton pulse systems, and so on.

\section{CONCLUSION}

A truly near zero ultra-flattened chromatic dispersion with high birefringence and low confinement loss HF has been proposed in a broad wavelength range. It has been shown through numerical simulation results that a five-ring HBDF-HF can assume nearly zero ultra-flattened dispersion of $0 \pm$ $0.5 \mathrm{ps} / \mathrm{nm} / \mathrm{km}$ in a 1.25 to $1.65 \mu \mathrm{m}$ (400 nm flat band) wavelength range with high birefringence of 0.0033 and low confinement losses of $0.0008 \mathrm{~dB} / \mathrm{km}$ at $1.55 \mu \mathrm{m}$. This proposed HBDF-HF design 
with highly birefringence nearly zero flattened chromatic dispersion and low confinement losses can be widely used for polarization control in fiber-optic sensors and optical communication systems. This fiber has a modest number of design parameters, five rings, two air-hole diameters, and a common air-hole pitch.

\section{REFERENCES}

[1] J. C. Knight, T. A. Birks, P. St. J. Russell, and D. M. Atkin, "All-silica single-mode optical fiber with photonic crystal cladding," Opt. Lett., vol. 21, pp.1547-1549 Oct. 1996.

[2] T. A. Birks, J. C. Knight, B. J. Mangan, and P. S. J. Russell, "Photonic crystal fibers: An endless variety," IEICE Trans. Electron., vol. E84-C, pp. 585-592, 2001.

[3] J. Broeng, D. Mogilevstev, S. E. Barkou, and A. Bjarklev, "Photonic crystal fibers: A new class of optical waveguides," Opt. Fiber Technol., vol. 5, pp. 305-330, 1999.

[4] S. M. A. Razzak, Y. Namihira, and F. Begum, "Ultra-flattened dispersion photonic crystal fiber," Electron. Lett., vol. 43, pp. 615-617, 2007.

[5] K. Saitoh, N. J. Florous, and M. Koshiba, "Ultra-flattened chromatic dispersion controllability using a defect-core photonic crystal fiber with low confinement loss,” Opt. Express, vol. 13, pp. 8365-8371, 2005.

[6] H. Ademgil and S. Haxha, "Highly Birefringent Photonic Crystal Fibers With Ultralow Chromatic Dispersion and Low Confinement Losses," J. Lightwave Technology, vol. 26,pp. 441-448, 2008.

[7] J. Ju, W. Jin, and M. S. Demokan, "Properties of a highly birefringent photonic crystal fiber," IEEE Photon. Technol. Lett., vol. 15, no. 10, pp. 1375-1377, Oct. 2003.

[8] T. Nasilowski, P. Lesiak, R. Kotynski, M. Antkowiak, A. Fernandez, F. Berghmans, and H. Thienpont, "Birefringent photonic crystal fiberas a multi parameter sensor," in Proc. Symp. IEEE, 2003, pp. 29-32.

[9] Y. Yue, G. Kai, Z. Wang, T. Sun, L. Jin, Y. Lu, C. Zhang, J. Liu, Y. Li, Y. Liu, S. Yuan, and X. Dong, "Highly birefringent elliptic-hole photonic crystal fibre with squeezed hexagonal lattice," Opt. Lett., vol. 32, pp. 469-471, 2007.

[10] A. Ferrando, E. Silvestre, P. Andres, J. J. Miret, and M. Andres, "Nearly zero ultraflattened dispersion in photonic crystal fibers," Opt. Lett., vol. 25, pp. 790-792, 2000.

[11] K. Saitoh, M. Koshiba, T. Hasegawa, E. Sasaoka, "Chromatic dispersion control in photonic crystal fibers: application to ultra-flattened dispersion,” Opt. Express, vol. 11, no. 8, pp.843-852, April 2003.

[12] K. Saitoh and M. Koshiba, "Full-vectorial imaginary-distance beam propagation method based on a finite element scheme: Application to photonic crystal fibers," IEEE J. Quantum Electron., vol. 38, no. 7, pp. 927-933, Jul. 2002.

[13] A. Ortigosa-Blanch, J. C. Knight, W. J. Wadsworth, J. Arriaga, B. J. Mangan, T. A. Birks, and P. S. J. Russell, "Highly birefringent photonic crystal fibers," Opt. Lett., vol. 25, pp. 1325-1327, 2000. 